\title{
Nature of spin Hall effect in a finite ballistic two-dimensional system with Rashba and Dresselhaus spin-orbit interaction
}

\author{
Yanxia Xing, ${ }^{1}$ Qing-feng Sun, ${ }^{1, *}$ and Jian Wang ${ }^{2}$ \\ ${ }^{1}$ Beijing National Laboratory for Condensed Matter Physics and Institute of Physics, Chinese Academy of Sciences, \\ Bejing 100080, China \\ ${ }^{2}$ Department of Physics and the Center of Theoretical and Computational Physics, The University of Hong Kong, \\ Pokfulam Road, Hong Kong, China
}

(Received 8 February 2006; revised manuscript received 12 April 2006; published 22 May 2006)

\begin{abstract}
The spin Hall effect in a finite ballistic two-dimensional system with Rashba and Dresselhaus spin-orbit interaction is studied numerically. We find that the spin Hall conductance is very sensitive to the transverse measuring location, the shape and size of the device, and the strength of the spin-orbit interaction. Not only the amplitude of spin Hall conductance, but also its sign, can change. This nonuniversal behavior of the spin Hall effect is essentially different from that of the charge Hall effect, in which the Hall voltage is almost invariant with the transverse measuring site and is a monotonic function of the strength of the magnetic field. This surprise behavior of the spin Hall conductance is attributed to the fact that the eigenstates of the spin Hall system are extended in the transverse direction and do not form the edge states.
\end{abstract}

DOI: 10.1103/PhysRevB.73.205339

\section{INTRODUCTION}

The Hall effect (from now on referred to as the charge Hall effect) is a well-known important phenomena in condensed-matter physics. It occurs due to the Lorentz force that deflects like-charge carriers towards one edge of the sample creating a voltage transverse to the direction of current. Recently, another interesting phenomena, the spin Hall effect (SHE), has been discovered and has attracted considerable attention. Here, spin accumulations emerge on the transverse sides of the sample when adding a longitudinal electric field or bias. If external leads are connected to the sides, the pure transverse spin current is generated. The SHE can either be extrinsic due to the spin-dependent scattering ${ }^{1,2}$ or intrinsic due to the spin-orbit (SO) interaction. The intrinsic SHE is predicted first by Murakami et al. and Sinova et al. in a Luttinger SO coupled three-dimensional (3D) $p$-doped semiconductor ${ }^{3}$ and a Rashba SO coupled twodimensional electron gas (2DEG) (Ref. 4), respectively. After that a number of recent works have focused on this interesting issue. ${ }^{5-19}$ For an infinite system, it was pointed out that the spin Hall conductivity is very sensitive to disorder, ${ }^{5-11}$ and the SHE vanishes even in a very weak disorder. On the other hand, in the finite mesoscopic ballistic system, the SHE can survive. ${ }^{12-18}$ By using the Landauer-Büttiker formalism and the tight-binding Hamiltonian ${ }^{20,21}$, the SHE and spin polarization have been studied in the dirty ${ }^{12-14}$ or clean ${ }^{15-17}$ mesoscopic samples. These investigations show that the SHE is still present below a critical disorder. Experimentally, the SHE is observed on $n$-type GaAs (Ref. 22) and on $p$-type GaAs (Ref. 23), where the transverse spin accumulations are detected by Kerr rotation spectroscopy or the circularly polarized light-emitting diode, respectively.

In this paper, we study the nature of the SHE in a finite 2DEG, and mainly focus on the comparison between the SHE and the charge Hall effect. This is because the SHE and the charge Hall effect are so analogous, intuitively they should have similar properties. In the charge Hall effect, the Hall voltage is a universal constant along the transverse
PACS number(s): 72.25.Dc, 71.70.Ej, 72.10.-d, 73.50.Jt

edge, i.e., it is independent of the transverse measuring location and the width of device. At least its sign is unchanged. Does the spin current possess similar universal behavior in SHE? The results are very surprising and show that the transverse spin current in the SHE is strongly dependent on the measuring location and the device's shape. ${ }^{24}$ Not only the intensity but also its sign can change. These results indicate that the SHE is not as clean as the charge Hall effect, and all the measured quantity in SHE are very sensitive on the details of the system. We attribute these nonuniversal behaviors to the extensive eigenstates in the transverse direction in the SHE.

\section{MODEL AND FORMULATION}

The system we considered is shown in Fig. 1, which consists of a finite central ballistic region attached to four semiinfinite leads. The Rashba and Dresselhaus SO interactions ${ }^{25}$ are present only in the central gray rectangular region with the size $N \times W$. In order to study the geometric effect, two zero-SO coupling (NSO) zones (central white regions in Fig. 1) with the size $N \times m$ are also patched. All the leads are

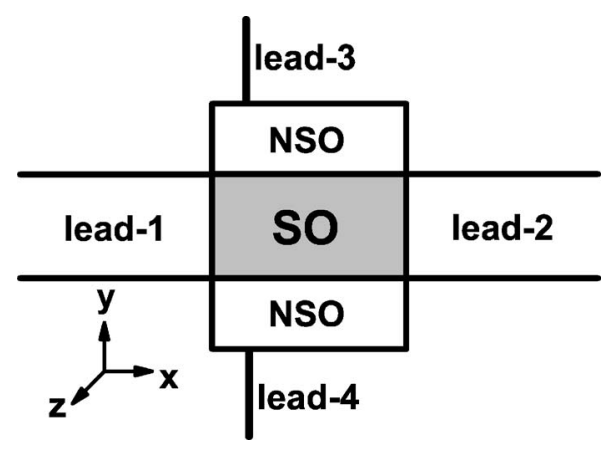

FIG. 1. Schematic diagram for the mesoscopic four-terminal device, in which the central gray region (marked by "SO") has the Rashba and Dresselhaus SO interactions, but two central white zones (marked by "NSO") are without the SO coupling. 

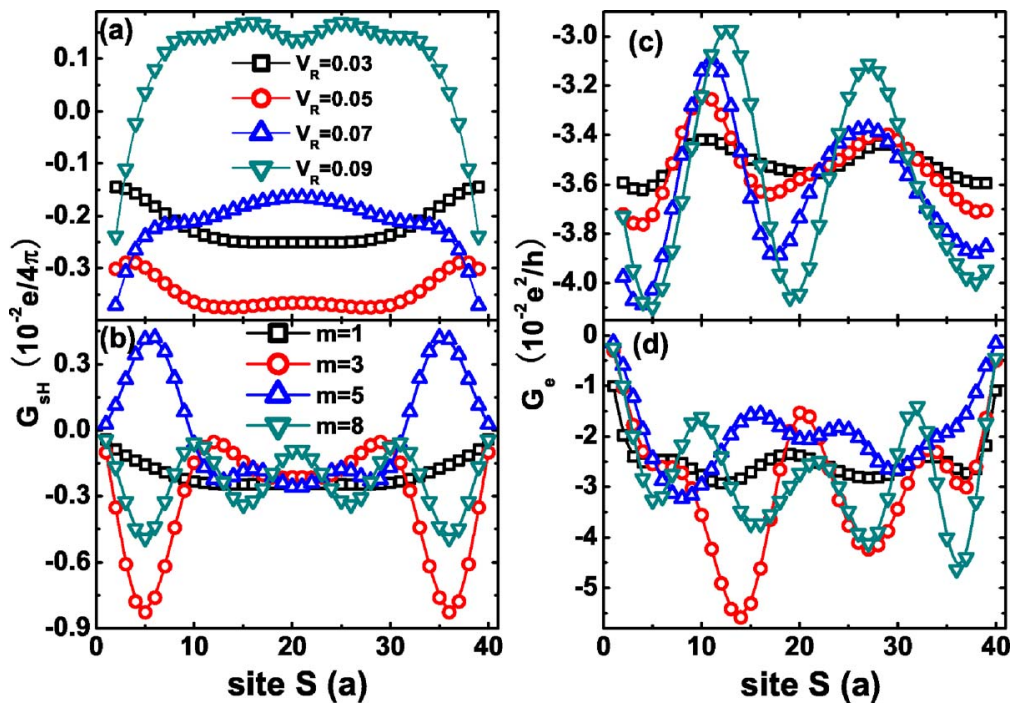

FIG. 2. (Color online) $G_{s H}$ and $G_{e}$ vs the measuring site $S$ for different $V_{R}=0.03,0.05,0.07$, and 0.09 with $m=0$ [panels (a) and (c)], or different width $m$ of the NSO's zone: $m=1,3,5$, and 8 with $V_{R}=0.03$ [panels (b) and (d)]. The other parameters are $V_{D}=0, N=40$, and $W=30$. assumed to be clean and ideal, without any SO coupling. The two longitudinal leads (lead-1 and lead-2) have the width $W$, which is the same as the width of the central SO region. On the other hand, in order to study the local spin Hall conductance and its dependence on the measuring location, two transverse leads (lead-3 and lead-4) are assumed to be one dimensional (1D) with the width 1 , and they can be coupled to any edge location $S$ along the $x$ direction.

The above system can be described by the Hamiltonian $H_{0}=p^{2} / 2 m^{*}+V(x, y)+\alpha\left(\sigma_{x} p_{y}-\sigma_{y} p_{x}\right)+\beta\left(\sigma_{x} p_{x}-\sigma_{y} p_{y}\right)$, where $\alpha$ and $\beta$ are the coefficients of the Rashba and Dresselhaus SO interactions. ${ }^{25}$ Then in the tight-binding representation, this Hamiltonian can be written as: ${ }^{12,13}$

$$
\begin{aligned}
H= & \sum_{i}\left[a_{i \uparrow}^{\dagger} a_{i \downarrow}^{\dagger}\right]\left[\begin{array}{cc}
-t & -i V_{D}+V_{R} \\
-i V_{D}-V_{R} & -t
\end{array}\right]\left[\begin{array}{c}
a_{i+\delta_{x} \uparrow} \\
a_{i+\delta_{x} \downarrow}
\end{array}\right] \\
& +\sum_{i}\left[a_{i \uparrow}^{\dagger} a_{i \downarrow}^{\dagger}\right]\left[\begin{array}{cc}
-t & -i V_{R}+V_{D} \\
-i V_{R}-V_{D} & -t
\end{array}\right]\left[\begin{array}{l}
a_{i+\delta_{y} \uparrow} \\
a_{i+\delta_{y} \downarrow}
\end{array}\right]+\text { H.c. },
\end{aligned}
$$

where $t=\hbar^{2} / 2 m^{*} a^{2}$ is the hopping matrix element with the lattice constant $a$. In order for the bandwidth of the 1D lead-3 and lead- 4 to be in the same range of $-4 t$ to $4 t$, the hopping matrix element in these two leads is set to be $2 t$. Here $V_{R}=\hbar \alpha / 2 a$ and $V_{D}=\hbar \beta / 2 a$ represent the strength of the Rashba and Dresselhaus interactions, respectively, and $V_{R}$ and $V_{D}$ are nonzero only in the central gray region. $\delta_{x}$ and $\delta_{y}$ in Eq. (1) are the unit vectors along the $x$ and $y$ directions.

Since there is no SO interaction in the leads, the spin $\sigma$ in the leads is a good quantum number and the definition of the spin current is unambiguous. Then the particle current $I_{p \sigma}$ in the lead- $p(p=1,2,3$, and 4) with spin index $\sigma(\sigma=\uparrow$, or $\downarrow$ stands for the $+z$ or $-z$ direction) can be obtained from the Landauer-Büttiker formula: $I_{p \sigma}=(e / h) \sum_{q \neq p, \sigma^{\prime}} T_{p \sigma, q \sigma^{\prime}}\left(V_{p}\right.$ $-V_{q}$ ) (Refs. 20 and 21), where $V_{p}$ is the bias in the lead- $p$ and $T_{p \sigma, q \sigma^{\prime}}$ is the transmission coefficient from the lead- $q$ with spin $\sigma^{\prime}$ to the lead- $p$ with spin $\sigma$. The transmission coefficient can be calculated from $T_{p \sigma, q \sigma^{\prime}}$
$=\operatorname{Tr}\left[\Gamma_{p \sigma} G^{r} \Gamma_{q \sigma^{\prime}} G^{a}\right]$, where the linewidth function $\Gamma_{p \sigma}$ $=i\left(\Sigma_{p \sigma}^{r}-\Sigma_{p \sigma}^{r \dagger}\right)$, the Green's function $G^{r}=\left[G^{a}\right]^{\dagger}=\left\{E_{F}-H_{0}\right.$ $\left.-\Sigma_{p \sigma} \Sigma_{p \sigma}^{r}\right\}^{-1}$ (Ref. 20), and $\Sigma_{p \sigma}^{r}$ is the retarded self-energy. After solving $I_{p \sigma}$, the spin current $I_{p}^{s}$ and the charge current $I_{p}^{e}$ can be obtained straightforwardly: $I_{p}^{e}=e\left\{I_{p \uparrow}+I_{p \downarrow}\right\}$ and $I_{p}^{s}$ $=(\hbar / 2)\left\{I_{p \uparrow}-I_{p \downarrow}\right\}$. The terminal voltages $V_{p}$ are set as: $V_{1}=V$ and $V_{2}=0$, i.e., a longitudinal bias $V$ is added between the lead- 1 and the lead-2. The transverse lead-3 and lead-4 act as the voltage probes, and their voltages $V_{3}$ and $V_{4}$ are calculated from the condition $I_{3}^{e}=I_{4}^{e}=0$. Then the transverse spin Hall conductances are: $G_{3 s H}=I_{3}^{s} / V_{1}$ and $G_{4 s H}=I_{4}^{s} / V_{1}$. For comparison, we also calculate the transverse charge currents or the charge conductances $\left(G_{3 e}=I_{3}^{e} / V_{1}\right.$ and $\left.G_{4 e}=I_{4}^{e} / V_{1}\right)$ in the same device but under a different condition $V_{3}=V_{4}=0$ instead of $I_{3}=I_{4}=0$. In the numerical calculation, we take $E_{F}=-3.8 t$ which is near the band bottom $-4 t$, and $t=1$ as an energy unit, then the corresponding lattice constant $a$ $\approx 3 \mathrm{~nm}$ (Ref. 18). The device's sizes (i.e., $N, W$, and $m$ ) are chosen in the same order with the spin precession length $L_{S O}$ over the precessing angle $\pi$. Here $L_{S O}=\pi a t / 2 V_{R}$. If taking $V_{R}=0.03 t$, then $L_{S O} \approx 50 a$.

\section{NUMERICAL RESULTS AND DISCUSSION}

First, we consider the case that the center region has only Rashba interaction $V_{R}\left(V_{D}=0\right)$ and two NSO zones do not exist with $m=0$. While $V_{D}=0$, it can be shown that $G_{3 s H}=$ $-G_{4 s H} \equiv-G_{s H}$ and $G_{3 e}=G_{4 e} \equiv G_{e}$. The spin Hall conductance $G_{s H}$ versus the measuring site $S$ and $V_{R}$ are depicted in Figs. 2(a) and 3(a). We see that $G_{s H}$ depends on the location of measuring sites $S$, and it can even change its sign, e.g., when $V_{R}=0.09$ [see Fig. 2(a)]. On the other hand, at the fixed measuring site with different $V_{R}$, the curve of $G_{s H}$ versus $V_{R}$ can also cover the range from negative to positive [see Fig. 3(a)]. In contrast to the charge Hall effect, their behaviors are essentially different. The Hall voltage or the charge Hall conductance usually is a monotonously increasing function of the strength of the external magnetic field. Furthermore, both usually are unchanged with the transverse measuring sites. 


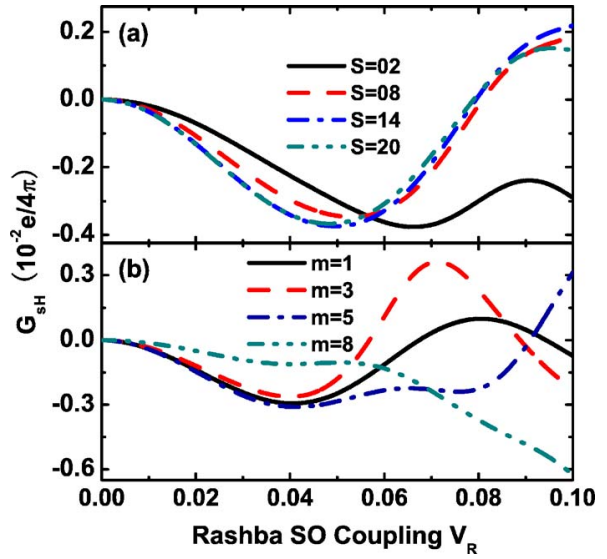

FIG. 3. (Color online) $G_{s H}$ vs $V_{R}$ for different site $S$ with $m$ $=0$ [panel (a)], or different width $m$ with $S=20$ [panel (b)]. The other parameters are the same as Fig. 2.

Next, we attach two NSO zones to the system (see Fig. 1). For the charge Hall effect, the charge accumulates in the transverse boundaries. If two zones having no magnetic field are attached, the charge accumulation will naturally transfer from the original boundaries to the new one; as a result the Hall voltage and the charge Hall conductance do not change much. How is the spin Hall conductance $G_{s H}$ affected when two NSO zones are attached? Figures 2(b) and Fig. 3(b) show, respectively, $G_{s H}$ versus the sites $S$ and $V_{R}$ for different thickness $m$ of the NSO zone. The results show that the spin Hall conductance $G_{s H}$ is strongly affected by the NSO zones. For example, in the curves $G_{s H^{-}} S$, for $m=0$ or 1 (see Fig. 2) $G_{s H}$ is flat, and it is negative at all site $S$. With increasing $m$, $G_{s H}$ shows an oscillation behavior. In particular, $G_{s H}$ can be positive, i.e., change its sign, for some value of $m$ (e.g., $m$ $=5)$. In the curve of $G_{s H}$ versus $V_{R}$ it also exhibits the similar results that $G_{s H}$ is strongly dependent on $m$ including changing its sign [see Fig. 3(b)].

For comparison, we also show the charge conductance $G_{e}$ for the same system but different bias conditions $V_{2}=V_{3}$ $=V_{4}=0$ [see Figs. 2(c) and 2(d)]. We see that $G_{e}$ is always negative and exhibits an oscillation behavior. For $m=0$ (i.e., without the NSO zones), $G_{e}$ is weakly dependent on the site
$S$, whereas for $m \neq 0$, the oscillatory amplitude of $G_{e}$ increases slightly. In particular, the charge conductance $G_{e}$ is nearly ten times larger than the spin Hall conductance $G_{s H}$. This also means $G_{s H}$ is much smaller than the universal value $\mathrm{Ne} / 4 \pi$, where $N$ is the channel number and $N=2$ in the present device, because the lead-3's and lead-4's width is 1 . Notice that in the charge Hall effect, the Hall conductance usually takes the universal value $\mathrm{Ne}^{2} / h$.

Let us study the spin Hall conductance $G_{s H}$ versus the transverse width $W$ of the center SO's regions. $G_{s H}$ and $G_{e}$ versus $W$ exhibit almost periodic peaks [see Figs. 4(a) and 4(c)]. Note that the cutoff energy of the subband (i.e., the transverse energy levels) are about $n^{2} \hbar^{2} \pi^{2} / 2 m W^{2}$, which shifts down with increasing the width $W$. For the Fermi level $E_{F}$ across a subband, a jump emerges in the curves of $G_{s H^{-}} W$ (or $G_{e}-W$ ), due to the large density of state near the band edge. As a result for a given period (e.g., $W=44,45,46$, and 47), $G_{s H}$ and $G_{e}$ versus the site $S$ [see Figs. 4(b) and 4(d)], exhibit the oscillation behavior. As the Fermi level across the subband edge $(W=47), G_{s H}$ can change its sign while $G_{e}$ is always negative.

In the following, we investigate the case when the Dresselhaus SO interaction is present, i.e., $V_{D} \neq 0$. As mentioned above, at $V_{D}=0$ the spin currents through the lead-3 and lead-4 are conserved, i.e., $G_{3 s H}=-G_{4 s H}$. However, when $V_{D} \neq 0$ and $V_{R} \neq 0, G_{3 s H} \neq-G_{4 s H}$. On the other hand, the spin Hall conductance has the symmetry with $G_{3 s H}(S)$ $=-G_{4 s H}(W-S)$ due to the symmetry of our system. It is worth to point out when $V_{D}=V_{R}, G_{4 s H}=G_{3 s H}=0$, which is similar with the Ref. 14. In Fig. 5, $G_{4 s H}$ versus the site $S$ for different $V_{R}$ or different width $m$ of the NSO zone is plotted. Here $G_{4 s H}$ exhibits similar characters as in the case of $V_{D}$ $=0: G_{4 s H}$ is very sensitive to the transverse measuring site $S$, and it can even change its sign (e.g., $V_{R}=0.07,0.09$ ). While $m \neq 0, G_{4 s H}$ oscillates with the site $S$ along with the variation of its sign. All these behaviors are in contrast to the charge Hall conductance.

Finally, we emphasize that the sensitivity of spin Hall conductance to the location of measuring sites is a generic feature not due to the 1D nature of the lead-3 and lead-4. We have performed similar calculations when the widths of lead-3 and lead-4 are 3 and 5. The conclusion remains. In
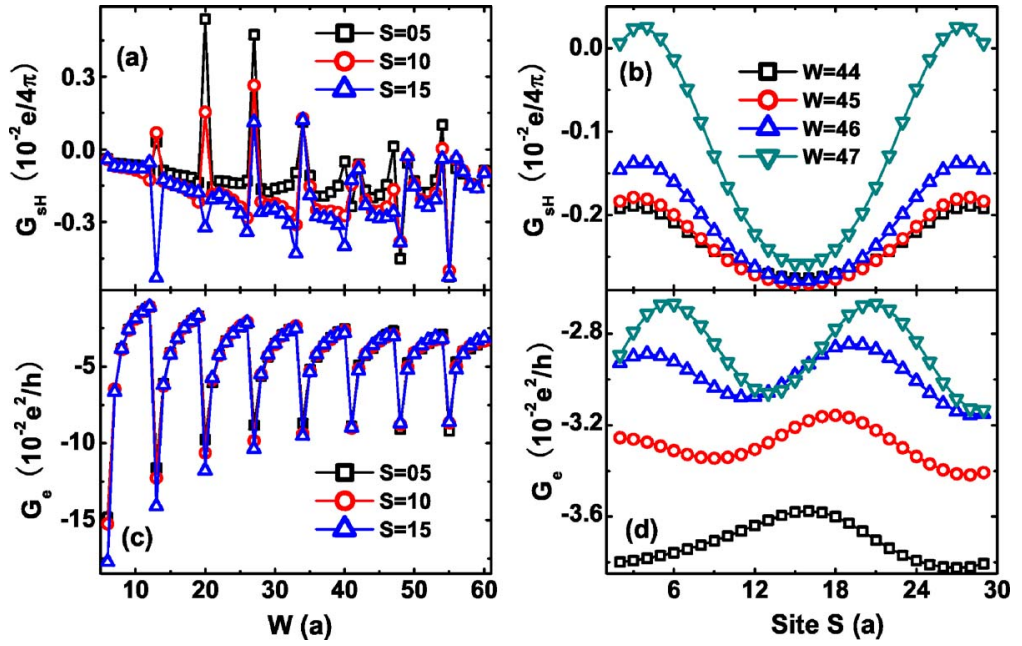

FIG. 4. (Color online) Left panel: $G_{s H}$ (a) and $G_{e}$ (c) vs the transverse width $W$ for the site $S$ $=5,10$, and 15. Right panel: $G_{s H}(\mathrm{~b})$ and $G_{e}(\mathrm{~d})$ vs the site $S$ for different transverse width $W$ $=44,45,46$, and 47. The other parameters are $V_{R}=0.03, V_{D}=0, N=30$, and $m=0$. 


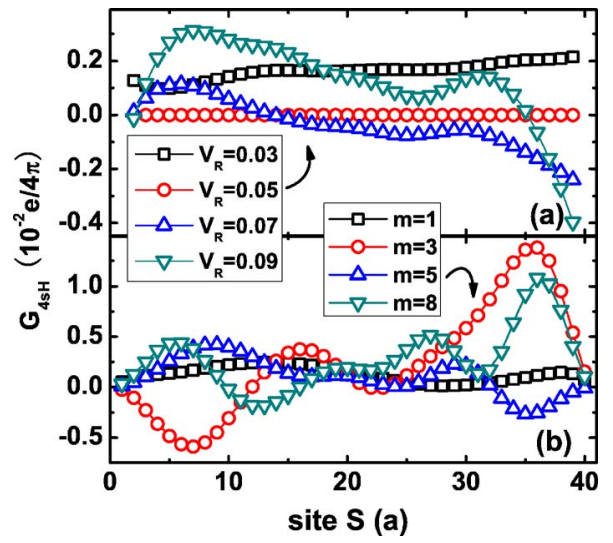

FIG. 5. (Color online) $G_{4 s H}$ vs the measuring site $S$ for different Rashba SO coupling strength $V_{R}=0.03,0.05,0.07$, and 0.09 with $m=0$, or different width of the NSO's zone: $m=1,3,5$, and 8 with $V_{R}=0.03$. The other parameters are $V_{D}=0.05, N=40$, and $W=30$.

addition, if the lead-3 and lead-4 are placed at two different measuring sites along the $x$ direction, $G_{3 s H}$ and $G_{4 s H}$ are affected even stronger.

Why are the characters of the SHE so different with the charge Hall effect? Why is the spin Hall conductance $G_{s H}$ so sensitive (even its sign) to the measurement site $S$, the shape of device, and so on? We attribute them to the following two reasons. (1) In the quantum Hall effect the edge states emerge and play an important role. However, for a system that exhibits SHE, e.g., the quasi-1D quantum wire having Rashba SO interaction, its eigenstates are extended in the transverse direction and they do not form edge states. ${ }^{26}(2)$ The force in the charge Hall effect always points to a specific direction, e.g., $+y$. But the force in the SHE is dependent on the spin $\sigma$, and its sign can vary. ${ }^{27}$

In summary, the spin Hall conductance is strongly dependent on the transverse measuring site, the device's shape, and the strength of the spin-orbit interaction. Not only the magnitude, but also its sign, can change. These characters are very different from that of the charge Hall effect, and the spin Hall conductance is not universal as the charge Hall conductance.

\section{ACKNOWLEDGMENTS}

We gratefully acknowledge helpful discussions with $\mathrm{H}$. Guo. This work was supported by NSF-China under Grants Nos. 90303016, 10474125, and 10525418. J.W. is supported by RGC Grant (No. HKU 7044/05P) from the government SAR of Hong Kong.
*Electronic address: sunqf@aphy.iphy.ac.cn

${ }^{1}$ J. E. Hirsch, Phys. Rev. Lett. 83, 1834 (1999).

${ }^{2}$ M. I. Dyakonov and V. I. Perel, JETP Lett. 13, 467 (1971); Phys. Lett. 35A, 459 (1971).

${ }^{3}$ S. Murakami, N. Nagaosa, and S. C. Zhang, Science 301, 1348 (2003); Phys. Rev. B 69, 235206 (2004).

${ }^{4}$ J. Sinova, D. Culcer, Q. Niu, N. A. Sinitsyn, T. Jungwirth, and A. H. MacDonald, Phys. Rev. Lett. 92, 126603 (2004).

${ }^{5}$ J. I. Inoue, G. E. W. Bauer, and L. W. Molenkamp, Phys. Rev. B 70, 041303(R) (2004).

${ }^{6}$ E. G. Mishchenko, A. V. Shytov, and B. I. Halperin, Phys. Rev. Lett. 93, 226602 (2004).

${ }^{7}$ E. I. Rashba, Phys. Rev. B 70, 201309(R) (2004).

${ }^{8}$ Y. Yao and Z. Fang, Phys. Rev. Lett. 95, 156601 (2005); G. Y. Guo, Y. Yao, and Q. Niu, ibid. 94, 226601 (2005).

${ }^{9}$ Z. F. Jiang, R. D. Li, S.-C. Zhang, and W. M. Liu, Phys. Rev. B 72, 045201 (2005).

${ }^{10}$ O. Chalaev and D. Loss, Phys. Rev. B 71, 245318 (2005).

${ }^{11}$ B. A. Bernevig and S. C. Zhang, Phys. Rev. Lett. 95, 016801 (2005); R. Raimondi and P. Schwab, Phys. Rev. B 71, 033311 (2005).

${ }^{12}$ L. Sheng, D. N. Sheng, and C. S. Ting, Phys. Rev. Lett. 94, 016602 (2005); L. Sheng, D. N. Sheng, C. S. Ting, and F. D. M. Haldane, Phys. Rev. Lett. 95, 136602 (2005).

${ }^{13}$ C. P. Moca and D. C. Marinescu, Phys. Rev. B 72, 165335 (2005).

${ }^{14}$ J. Li, L. Hu, and S.-Q. Shen, Phys. Rev. B 71, 241305(R) (2005).

${ }^{15}$ E. M. Hankiewicz, L. W. Molenkamp, T. Jungwirth, and J. Sinova, Phys. Rev. B 70, 241301(R) (2004).

${ }^{16}$ B. K. Nikolić, L. P. Zârbo, and S. Souma, Phys. Rev. B 72, 075361 (2005).

${ }^{17}$ A. Reynoso, G. Usaj, and C. A. Balseiro Phys. Rev. B 73, 115342
(2006).

${ }^{18}$ B. K. Nikolić, S. Souma, L. P. Zârbo, and J. Sinova, Phys. Rev. Lett. 95, 046601 (2005); J. Yao and Z. Q. Yang, Phys. Rev. B 73, 033314 (2006); J. Wang, K. S. Chan, and D. Y. Xing, ibid. 73, 033316 (2006).

${ }^{19}$ J. Schliemann and D. Loss, Phys. Rev. B 71, 085308 (2005); X. Dai, Z. Fang, Y.-G. Yao, and F.-C. Zhang, Phys. Rev. Lett. 96, 086802 (2006).

${ }^{20}$ Electronic Transport in Mesoscopic Systems, edited by S. Datta (Cambridge University Press, 1995), Chaps. 2 and 3.

${ }^{21}$ T. P. Pareek, Phys. Rev. Lett. 92, 076601 (2004).

${ }^{22}$ Y. K. Kato, R. C. Myers, A. C. Gossard, and D. D. Awschalom, Science 306, 1910 (2004); V. Sih, R. C. Myers, Y. K. Kato, W. H. Lau, A. C. Gossard, and D. D. Awschalom, Nat. Phys. 1, 31 (2005).

${ }^{23}$ J. Wunderlich, B. Kaestner, J. Sinova, and T. Jungwirth, Phys. Rev. Lett. 94, 047204 (2005).

${ }^{24}$ The spin accumulation is also strongly influenced by boundary conditions for both extrinsic and intrinic SHE, which has been reporter in two recent works: W.-K. Tse, J. Fabian, I. Zutic, and S. Das Sarma, Phys. Rev. B 72, 241303(R) (2005); V. M. Galitski, A. A. Burkov, and S. Das Sarma, cond-mat/0601677 (unpublished).

${ }^{25}$ Y. A. Bychkov and E. I. Rashba, J. Phys. C, 17, 6039 (1984); G. Dresselhaus, Phys. Rev. 100, 580 (1955).

${ }^{26}$ In fact, the system of the quasi-1D quantum wire having Rashba SO interaction can exactly be solved, and its eigenstates indeed are extended in the transverse direction, e.g., see Q.-f. Sun and X. C. Xie, cond-mat/0505517 (unpublished).

${ }^{27}$ B. K. Nikolic, L. P. Zarbo, and S. Welack, Phys. Rev. B 72, 075335 (2005); S.-Q. Shen, Phys. Rev. Lett. 95, 187203 (2005); K. Yu. Bliokh, cond-mat/0511146 (unpublished). 